\title{
HIPERTENSÃO RESISTENTE EM PACIENTE COM DIAGNÓTICO DE HIPERALDOSTERONISMO PRIMÁRIO: RESPOSTA AO EPLERENONE
}

\author{
RESISTANT HYPERTENSION IN A PATIENT WITH PRIMARY \\ HYPERERALDOSTERONISM DIAGNOSIS: RESPONSE TO EPLERENONE
}

Antônio Carlos de Souza Spinelli', Rui Manuel dos Santos Póvoa², Julizar Dantas³

\section{RESUMO}

A maioria dos pacientes com hipertensão arterial (HA) não tem etiologia clara e é classificada como hipertensão primária. No entanto, $5 \%$ a 10\% desses pacientes podem ter hipertensão secundária, o que indica presença de uma causa subjacente e potencialmente reversível. Em adultos com 65 anos ou mais, estenose da artéria renal aterosclerótica, insuficiência renal e hipotireoidismo são causas comuns. A hipertensão secundária deve ser considerada na presença de sintomas e sinais sugestivos, como hipertensão grave ou resistente, idade de início inferior a 30 anos (especialmente antes da puberdade), hipertensão maligna ou acelerada e aumento agudo da pressão arterial a partir de leituras previamente estáveis. Outras causas subjacentes da hipertensão secundária incluem hiperaldosteronismo, apneia obstrutiva do sono, feocromocitoma, síndrome de Cushing, doença da tireoide, coarctação da aorta e uso de certos medicamentos. A hipertensão arterial resistente (HAR) é definida quando a pressão arterial (PA) permanece acima das metas recomendadas com o uso de três anti-hipertensivos de diferentes classes, incluindo um bloqueador do sistema renina-angiotensina (inibidor da enzima conversora da angiotensina [IECA] ou bloqueador do receptor de angiotensina [BRA]), um bloqueador dos canais de cálcio (BCC) de ação prolongada e um diurético tiazídico (DT) de longa ação em doses máximas preconizadas e toleradas, administradas com frequência, dosagem apropriada e comprovada adesão. Hipertensão arterial acompanhada de supressão da atividade da renina plasmática (ARP) e aumento da excreção de aldosterona caracteriza a síndrome de aldosteronismo primário. Esse quadro foi descrito, pela primeira vez em 1955 por Conn, em um paciente hipertenso grave hipocalêmico e com secreção elevada de aldosterona, que submetido à adrenalectomia direita resultou em cura da HA.

Descritores: Hipertensão Resistente; Hiperaldosteronismo Primário; Tratamento Farmacológico; Relato de Caso.

\begin{abstract}
Patients with arterial hypertension have no clear etiology and are classified as primary hypertension. However, 5\% to 10\% of these with hypertension may have the secondary form of disease, which indicates the presence of an underlying and potentially reversible cause. In adults aged 65 and over, the common causes of secondary hypertension are atherosclerotic renal artery stenosis, renal failure and hypothyroidism. Secondary hypertension should be considered in the presence of suggestive symptoms and signs, such as severe or resistant hypertension, age at onset less than 30 years (especially before puberty), malignant or accelerated hypertension and acute increase in blood pressure from previously stable readings. Other underlying causes of secondary hypertension include hyperaldosteronism, obstructive sleep apnea, pheochromocytoma, Cushing's syndrome, thyroid disease, coarctation of the aorta and use of others medications. Resistant arterial hypertension is defined when blood pressure remains above the recommended targets with the use of three antihypertensives of different classes, including a blocker of the renin-angiotensin system (inhibitor of the angiotensin-converting enzyme or angiotensin receptor blocker), a calcium channel blocker and a thiazide diuretic in maximum recommended and tolerated doses, administered frequently, appropriate dosage and proven adherence. Arterial hypertension accompanied by suppression of plasma renin activity and increased aldosterone excretion characterizes the primary aldosteronism syndrome. This condition was described in 1955 by Conn, in a severe hypokalemic hypertensive patient with high aldosterone secretion, who underwent right adrenalectomy resulted in a cure for the hypertension.
\end{abstract}

Keywords: Resistant Hypertension; Primary hyperaldosteronism; Pharmacological treatment; Case report.

1. Cardiocentro Natal, RN, Brasil.

2. Universidade de São Paulo UNIFESP São Paulo SP Brasil

3. Fundação Educacional Lucas Machado (FELUMA)- FCMMG Belo Horizonte MG Brasil

Correspondência: Antônio Carlos de Souza Spinelli. Rua Campos Sales, 742 Tirol. 5902O-300. Natal, RN, Brasil. spinelli@cardiol.br

http://dx.doi.org/10.47870/1519-7522/2021280144-7 


\section{INTRODUÇÃO}

Nas últimas décadas trabalhos publicados revelaram que níveis elevados de aldosterona também estão associados à hipertrofia ventricular esquerda, lesão renal, doença vascular ${ }^{1-3}$ e alterações estruturais e funcionais das artérias de médio calibre, ${ }^{4}$ assim como a lesões da microcirculação. ${ }^{5-7}$

Anteriormente era considerada forma rara de HA secundária (1\%), na atualidade parece estar presente em $22 \%$ dos pacientes com HAR. ${ }^{8,9}$ Gordon et al. observaram entre 52 hipertensos normocalêmicos em tratamento com anti-hipertensivos submetidos à quantificação da relação aldosterona - atividade da renina plasmática confirmada por medidas repetidas e por testes de supressão da aldosterona, que a incidência de aldosteronismo primário na população de hipertensos primários está entre 5 a 15\%, provavelmente, cerca de $12 \%$. ${ }^{10} \mathrm{~A}$ mesma conclusão chegou Lim et al. que demonstraram em população do Reino Unido que 1 em cada 10 pacientes hipertensos apresentava relação aldosterona - atividade da renina plasmática elevada sem necessariamente apresentar tumor adrenal (adenoma) ou hiperplasia. ${ }^{11}$ O adenoma da adrenal é a causa mais frequente, enquanto a hiperplasia uni ou bilateral é menos detectada. Carcinomas, apesar de infrequentes, também podem ser responsáveis pela instalação da doença. As principais causas de HA que acompanham o excesso de secreção de mineralocorticoide estão categorizadas com base nos níveis de renina e aldosterona.

\section{RELATO DE CASO}

Paciente do sexo masculino, 72 anos, com diagnóstico de hiperaldosteronismo primário desde 2013, evoluindo com hipocalemia e hipertensão arterial resistente.
Em 2013, foi submetido a TC do abdome com detecção da presença de adenoma de suprarrenal esquerda, sendo submetido ao cateterismo de veias renais em 25/10/2013, no Hospital Vila da Serra com os seguintes resultados: Aldosterona basal, sangue periférico (RIE): $26 \mathrm{ng} / \mathrm{dL}$; Aldosterona basal, suprarrenal esquerda (RIE): 14,6 ng/dL; Aldosterona basal, suprarrenal direita (RIE): 25,2 ng/dL. Desde então optou-se pelo tratamento conservador, incluindo-se a espironolactona $50 \mathrm{mg}$, IECA, BCC, betabloqueadores, diuréticos tiazídicos associados à amilorida. A introdução de espironolactona resultou em controle pressórico parcial, mantendo-se a hipocalemia, apesar dos níveis adequados da aldosterona. Aproximadamente 4 meses após o início do uso da espironolactona $50 \mathrm{mg}$, o paciente apresentou ginecomastia importante e extremanente dolorosa, optando-se pela redução da dose para $25 \mathrm{mg}$, que foi insuficiente para a manutenção do controle adequado da pressão arterial. $\mathrm{Na}$ evolução, o paciente apresentou edema angioneurótico secundário ao IECA, sendo substituído por BRA. Em novembro de 2019, durante o período de seguimento, o paciente fez a dosagem de Aldosterona: 14,68 ng/dL; Atividade de renina plasmática: $0,01 \mathrm{ng} / \mathrm{mL} / \mathrm{h}$; Relação A/R: 1468; Potássio: 2,7 mEq/La e ecodoppler de artérias renais sem apresentar alterações hemodinâmicas sugestivas de estenose de artérias renais (Quadro 1).

Em 2020, após interconsulta com endocrinologista e discussão do caso, concluímos pela manutenção do tratamento clínico e possibilidade de inclusão do eplerenone.

Apesar do uso de espironolactona $25 \mathrm{mg}$, indapamida $3 \mathrm{mg}+$ amilorida $6 \mathrm{mg}$, telmisartana $80 \mathrm{mg}$ BID, manidipino $10 \mathrm{mg}$ BID, Nebivolol 5 mg BID, e suplementação de $1.200 \mathrm{mg}$ de cloreto de potássio ao dia, persistiu a hipocalemia

Quadro 1. Aldosterona em amostra colhida na suprarrenal direita.

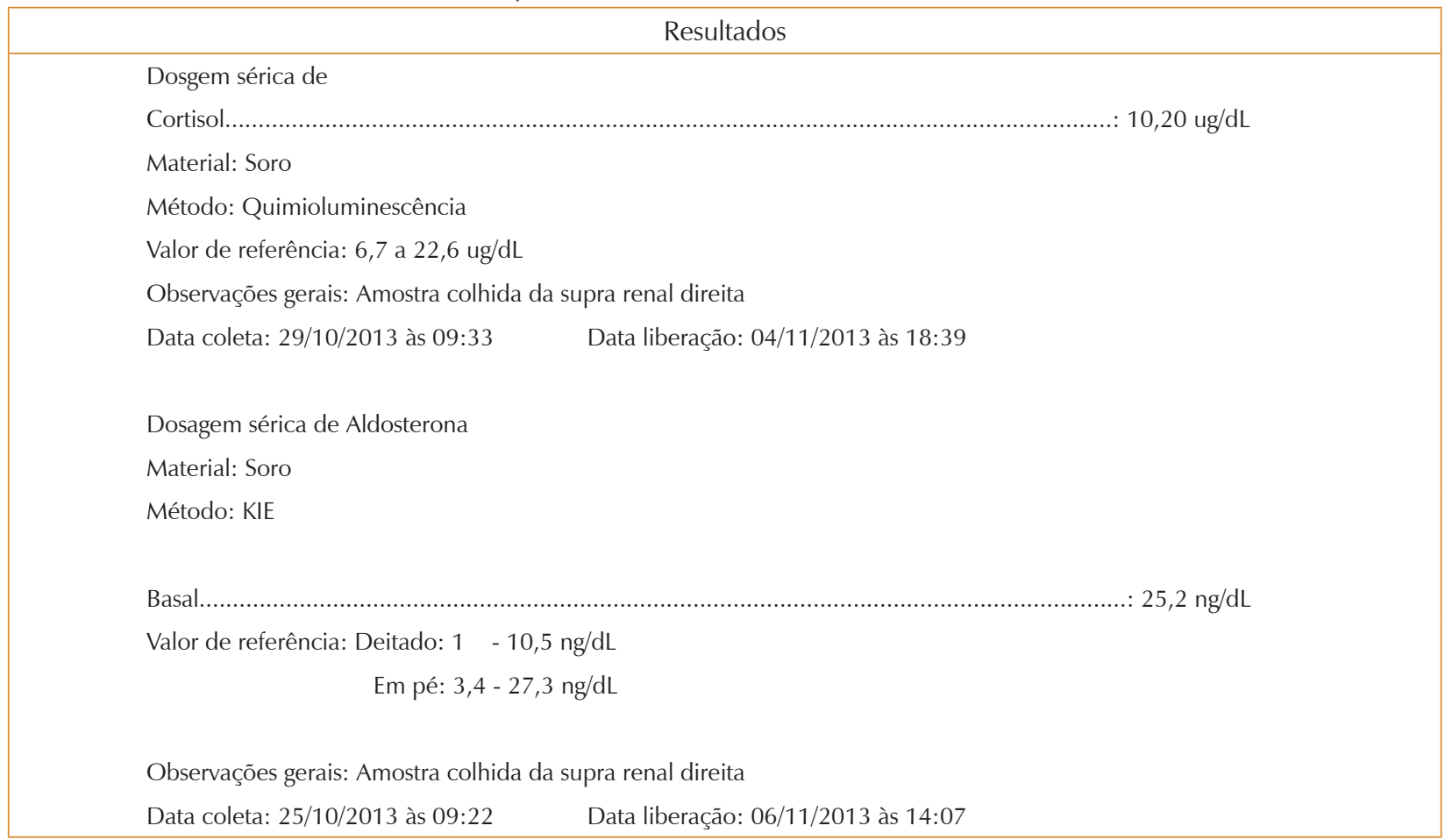


Quadro 2. Aldosterona em amostra colhida na suprarrenal esquerda.

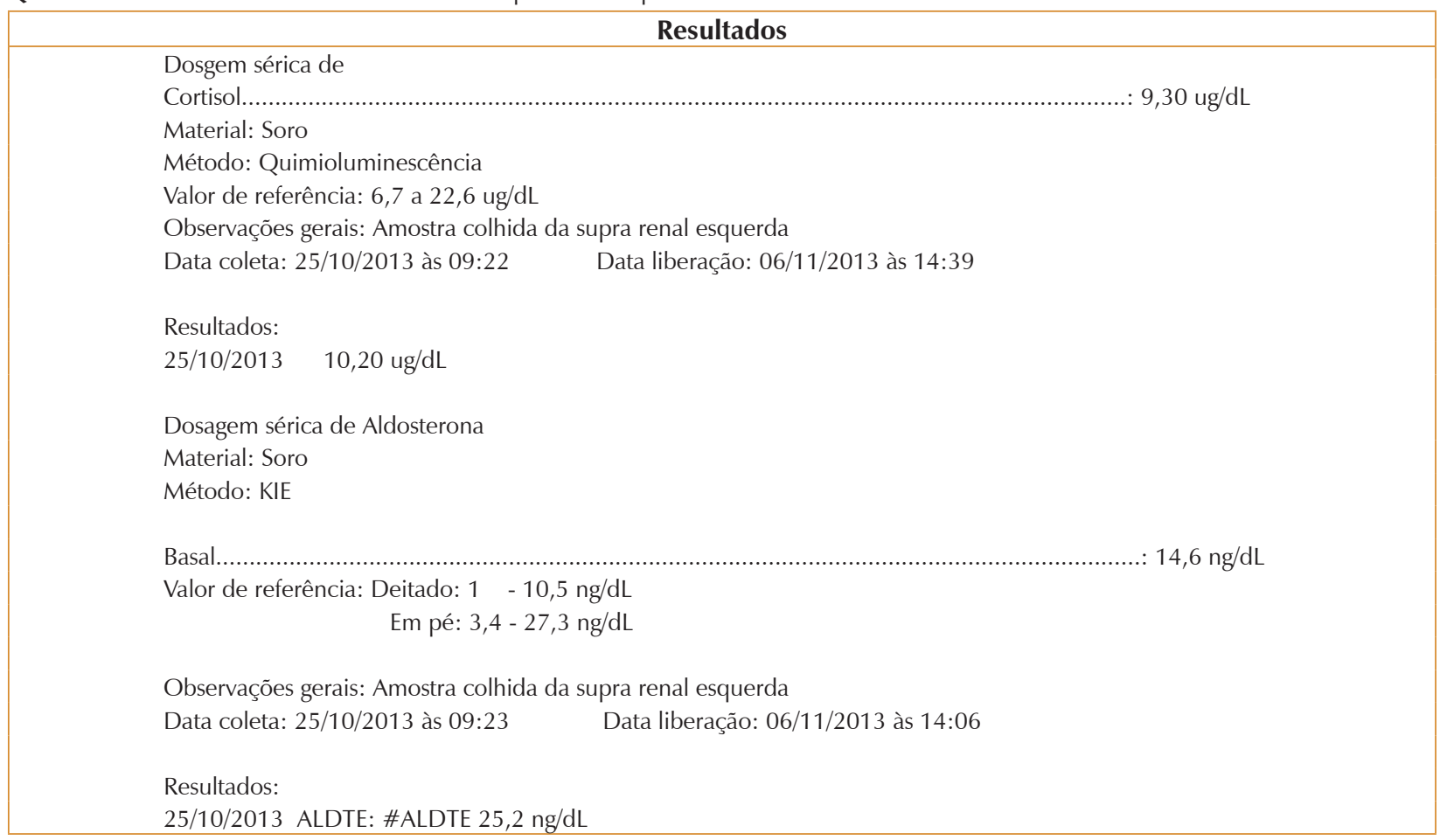

\begin{tabular}{|c|c|c|c|c|c|c|c|c|c|}
\hline \multicolumn{3}{|c|}{ MRPA ANTES DO EPLERENONE } & & & \multicolumn{3}{|c|}{ MRPA APÓS EPLERENONE } & \multirow[b]{2}{*}{ NOITE } & \multirow{3}{*}{ PAD } \\
\hline \multirow[t]{3}{*}{ DATA } & MANHÃ & & NOITE & & DATA & MANH & & & \\
\hline & PAS & PAD & PAS & PAD & & PAS & PAD & PAS & \\
\hline & $\mathrm{mmHg}$ & $\mathrm{mmHg}$ & $\mathrm{mmHg}$ & $\mathrm{mmHg}$ & & $\mathrm{mmHg}$ & $\mathrm{mmHg}$ & $\mathrm{mmHg}$ & $\mathrm{mmHg}$ \\
\hline MÉDIA & 195,4 & 100,3 & 186,6 & 93,4 & MÉDIA & 156,6 & 82,9 & 133,5 & 71,8 \\
\hline
\end{tabular}

Figura 3. MRPA antes e após o uso Eplerenone.

(2,4 mEq/L em 12/11/2020), e a MRPA confirmou HAR, com médias de PA: 195 x 100 mmHg pela manhã e 187 × 93 $\mathrm{mmHg}$ à noite, antes da ingestão dos medicamentos.

No início de dezembro de 2020, apresentou sintomas sugestivos de AIT, sendo submetido à angiotomografia computadorizara multíplice dos vasos cerebrais (09/12/2020) com resultado normal. Repetiu TC suprarrenais (09/12/2020), confirmando-se o adenoma suprarrenal $\mathrm{E}$, medindo 1,1 cm. Diante da HAR, e impossibilidade de manter a espironolactona na dosagem que permitisse a resposta terapêutica satisfatória, foi acrescentado o Eplerenone 50 mg, medicamento indisponível no Brasil, sendo importado da Holanda.

Após a administração do Eplerenone na dose de 50mg, em tomada única diária, houve redução das médias da pressão arterial obtidas pela MRPA: 156,6 x 82,9 mmHg pela manhã e 133,5 x 71,8 mmHg à noite (Figura ), e controle dos níveis séricos de potássio (4,0 mEq/L em 26/12/2020) nos limites fisiológicos.

Devido à dificuldade da importação do eplerenone em quantidade suficiente, ainda não foi possível ajustar a dose do medicamento. Atualmente foram suspensos a suplementação de potássio e reduzidas as doses de manidipino 10 mg e nebivolol 5 mg ao dia, mantendo-se os mesmos níveis tensionais.

\section{DISCUSSÃO}

No caso clínico apresentado, não se obteve o controle pressórico adequado avaliado pela MRPA (médias de PA: 195 x $100 \mathrm{mmHg}$ pela manhã e 187 x $93 \mathrm{mmHg}$ à noite), mesmo com o emprego de cinco fármacos em associação, ressaltando-se a limitação para o uso de doses plenas da espironolactona devido ao efeito colateral (ginecomastia sintomática).

O emprego de apenas 25 mg de espironolactona não foi suficiente para o controle da pressão arterial, nem para normalização dos níveis séricos da aldosterona e do potássio sérico.

Isso motivou a adição do eplerenone 50 mg em associação com os outros cinco fármacos, observando-se redução significativa da pressão arterial (MRPA com médias de PA: 156,6 x 82,9 mmHg pela manhã e 133,5 x 71,8 mmHg à noite) e normalização do potássio sérico após duas semanas do início do uso de eplerenone.

Importante ressaltar que na condução clínica deste caso, especialmente durante o período da pandemia de COVID-19, as medidas da pressão arterial fora do consultório foram realizadas em quase todos os períodos do seguimento com utilização da AMPA. A realização da MAPA foi um recurso utilizado apenas em anos anteriores, no início do acompanhamento. 


\section{CONCLUSÃO}

No presente caso, após o diagnóstico de hiperaldosteronismo primário, as medidas não farmacológicas e o tratamento farmacológico, incluindo o uso de espironolactona, foram adotados sem sucesso terapêutico, caracterizando a HAR. Durante o seguimento do paciente, concluiu-se pela manutenção do tratamento clínico, descartando-se a intervenção cirúrgica. Vários fármacos foram acrescentados à prescrição inicial sem controle adequado da pressão arterial. Uma resposta razoável ao tratamento só foi observada após o aumento da dose de espironolactona para 50 mg, resultando na ocorrência de ginecomastia sintomática, o que nos obrigou a buscar outra alternativa dentro da classe farmacológica dos antagonistas mineralocorticóides. A viabilização da importação do eplerenone por familiares do paciente nos permitiu associar $50 \mathrm{mg}$ de eplerenone com os outros cinco fármacos. Desde então, o paciente evoluiu com melhoria clínica, redução significativa da pressão arterial e normalização do potássio sérico, após duas semanas do uso do medicamento.

\section{REFERÊNCIAS}

1. Duprez DA, Bauwens FR, De Buyzere ML, et al. Influence of arterial blood pressure and aldosterone on left ventricular hypertrophy in moderate essential hypertension. Am J Cardiol. 1993;71(3): 17A-20A. doi: 10.1016/0002-9149(93)90240-d.

2. El-Gharbawy AH, Nadig VS, Kotchen JM, et al. Arterial pressure, left ventricular mass, and aldosterone in essential hypertension. Hypertension. 2001;37(3): 845-50. doi: 10.1161/01.hyp.37.3.845.

3. Dartsch T, Fischer R, GapelyukA, Weiergraeber M, Ladage D, Schneider T, et al. Aldosterone induces electrical remodeling independent of hypertension. Int J Cardiol. 2013; 164(2):170-8. doi: 10.1016/j.ijcard.2011.06.100.

4. Martinez-Aguayo A, Carvajal CA, Campino C, Aglony M, Bolte L, Garcia $\mathrm{H}$, et al., Primary aldosteronism and its impact on the generation of arterial hypertension, endothelial injury and oxidative stress. J Pediatr Endocrinol Metab. 2010; 23(4): 323-30. doi: 10.1515/jpem.2010.052.

5. Rocha R, Stier Jr CT, Kifor I, Ochoa-Maya MR, Rennke HG, Williams GH, et al. Aldosterone: a mediator of myocardial necrosis and renal arteriopathy. Endocrinology. 2000; 141(10): 3871-8. doi: 10.1210/endo.141.10.7711.

6. Rossi GP, BolognesiM, Rizzoni D, Seccia TM, Piva A, Porteri E, Tiberio GAM, et al. Vascular remodeling and duration of hypertension predict outcome of adrenalectomy in primary aldosteronism patients. Hypertension. 2008; 51(5): 1366-71. doi: 10.1161/HYPERTENSIONAHA.108.111369

7. Tsioufis C, Kyvelou S, Dimitriadis K, Syrseloudis D, Sideris S, Skiadas I, et al. The diverse associations of uric acid with low-grade inflammation, adiponectin and arterial stiffness in never-treated hypertensives. J Hum Hypertens. 2011; 25(9): 554-9. doi: 10.1038/jhh.2010.98. Epub 2010 Oct 21.

8. Calhoun DA. Is there an unrecognized epidemic of primary aldosteronism? Pro. Hypertension. 2007; 50(3): 447-53. doi: 10.1161/ HYPERTENSIONAHA.106.086116.

9. Kline GA, Prebtani APH, Leung AA, Schiffrin EL. Primary aldosteronism: a Common cause of resistant hypertension. CMAJ. 2017; 189(22): E773-E778. doi: 10.1503/cmaj.161486.

10. Gordon RD, Ziesak MD, Tunny TJ, Stowasser M, Klemm SA. Evidence that primary aldosteronism may not be uncommon: $12 \%$ incidence among antihypertensive drug trial volunteers. Clin Exp Pharmacol Physiol. 1993; 20(5): 296-8. doi: 10.1111/j.1440-1681.1993.tb01687.x.

11. Lim PO, Dow E, Brennan G, Jung RT, MacDonald TM. High prevalence of primary aldosteronism in the Tayside hypertension clinic population. J Hum Hypertens. 2000; 14(5): 311-5. doi: 10.1038/sj.jhh.1001013. 